\title{
Platelet-rich Plasma and Mesenchymal Stem Cells Local Infiltration Promote Functional Recovery and Histological Repair of Experimentally Transected Sciatic Nerves in Rats
}

Nikolaos Kokkalas ${ }^{1}$, Panagiotis Kokotis ${ }^{2}$, Kalliopi Diamantopoulou ${ }^{3}$, Antonios Galanos ${ }^{4}$, Pavlos Lelovas 5 , Dionysios J. Papachristou 6,7 , Ismene A. Dontas ${ }^{5}$, Ioannis K. Triantafyllopoulos ${ }^{8}$

1. Orthopaedics, Laboratory for Research of the Musculoskeletal System, KAT General Hospital, Medical School, National \& Kapodistrian University of Athens, Athens, GRC 2. Neurology, Laboratory of Clinical Neurophysiology, Aeginitio Hospital, Medical School, National \& Kapodistrian University of Athens, Athens, GRC 3. Pathology, KAT General Hospital, Athens, GRC 4. Epidemiology and Public Health, Laboratory for Research of the Musculoskeletal System, KAT General Hospital, Medical School, National \& Kapodistrian University of Athens, Athens, GRC 5. Veterinary Medicine, Laboratory for Research of the Musculoskeletal System, KAT General Hospital, Medical School, National \& Kapodistrian University of Athens, Athens, GRC 6. Pathology, Unit of Bone and Soft Tissue Studies, Laboratory of Anatomy-Histology-Embryology, School of Medicine, University of Patras, Patras, GRC 7. Pathology, School of Medicine, University of Pittsburgh, Pittsburgh, USA 8. Orthopaedics, HYGEIA Private Hospital, Athens, GRC

Corresponding author: Ioannis K. Triantafyllopoulos, sportdoc@otenet.gr

\section{Abstract}

\section{Introduction}

Platelet-rich plasma (PRP) products and mesenchymal stem cells (MSCs) seem to have a significant potential as neurogenic therapeutic modulator systems. This study aimed to investigate such biological blood derivatives that could enhance nerve regeneration when applied locally in the primary repair of peripheral nerve transection of an experimental rat model.

\section{Methods}

A total of 42 two-month-old male Wistar rats were divided into three "treatment" groups (control, PRP, and MSCs). All the subjects were operated under anesthesia, and the surgical site was infiltrated with either normal saline, PRP derived from the animal's peripheral blood, or MSCs derived from the animal's femoral bone marrow. All three groups were also sub-divided into two sub-groups based on the post-operative administration of Non-steroidal anti-inflammatory drugs (NSAIDs) or not in order to evaluate the effect of NSAIDs on the final outcome. Three months post-surgery, electromyography evaluation of both hind limbs (right operated and left non-operated) was performed. The animals were euthanized, and nerve repair specimens were prepared for histology.

Received 04/28/2020

Review began 05/05/2020 Review ended 05/13/2020 Published 05/24/2020

\section{() Copyright 2020}

Kokkalas et al. This is an open access article distributed under the terms of the Creative Commons Attribution License CC-BY 4.0., which permits unrestricted use, distribution, and reproduction in any medium, provided the original author and source are credited.

\section{Results}

PRP group had a significant effect $(\mathrm{p}<0.05)$ on the sciatic nerve repair when compared with the control group, whereas the MSC group had a positive effect but was not statistically significant $(\mathrm{p}=0.2)$. The number of counted neural axons at the area distal to the nerve repair site were significantly repetitive $(\mathrm{p}<0.05)$ in both the PRP and MSC groups when compared with the control group.

\section{Conclusions}

Both PRP and MSCs appear to play an essential role in the enhancement of nerve repair in terms of functionality and histology. MSCs group demonstrated a positive effect, whereas the PRP group showed statistically significant better results.

Categories: Neurosurgery, Orthopedics, Trauma

Keywords: plateler rich plasma, mesenchymal stem cells, nerve repair, nerve regeneration

\section{Introduction}

Side-to-side tension-free micro-surgical repair or transplantation of a nerve autograft to bridge a nerve gap remains the golden standard technique for the enhancement of the intrinsic regenerative potential of injured neuronal axons [1]. However, such treatments do not recreate the suitable cellular and molecular micro-environment for a satisfactory regeneration. Thus, recovery of such nerve injuries is incomplete [2].

In adjuvant biological treatment that would enhance nerve regeneration and improve nerve function, local application of platelet-rich plasma (PRP) derivatives and pure mesenchymal stem cells (MSCs) could be 
promising interventions in addition to the nerve repair. PRP products hold an important therapeutic potential as neuroprotective and neurogenic modulator systems [3]. In the literature, the results of PRP use are contradictory; there are studies supporting their boosting effects on nerve repair, whereas others put it in doubt [4].

MSCs, either derived from the bone marrow or adipose tissue, appear to enhance axon regeneration [5]. They produce this positive effect not only when delivered to the injured nerve or conduit bridging the nerve gap but also when administered intravenously. The MSCs' migration potential made their detection possible at the site of sciatic nerve injury on day 7 post-intravenous injection to mice and enhanced the functional recovery of the sciatic nerve [6,7]. However, this observation was not in agreement with the findings of another study in a rat sciatic nerve injury model, where MSCs combined with a fibrin glue conduit promoted axon regeneration only when exposed to immunosuppressive treatment with cyclosporine A [6].

Therefore, the impact of biological agents on nerve regeneration continues to be a field of interest. The aim of this experimental animal study was to show the effect of PRP and bone-derived MSCs (b-MSCs) on the enhancement of nerve regeneration when locally applied in the primary repair of peripheral sciatic nerve transection using an experimental animal model. The originality of this study is the comparison of electromyography (EMG) results between the operated leg and the non-operated one, as well as the comparison of the histopathological results of the injured nerve distal and proximal with respect to the repair. In addition, the role of intramuscularly administered NSAIDs on the final outcome of nerve repair was also evaluated.

\section{Materials And Methods}

The protocol was approved by the Directorate of Veterinary Services of Prefecture of Athens, Attica, Greece, according to Greek legislation regarding ethical and experimental procedures (EL 25 BIO 018). Forty-two two-month-old male Wistar albino rats weighing between 200 and $240 \mathrm{~g}$ were included in the study. The rats were randomly divided into three "treatment" groups: (1) control group, where nerve repair was only performed, (2) the PRP group, where autologous PRP was isolated from the peripheral vein blood and locally infiltrated into the nerve repair, and (3) the MSCs group, where b-MSCs obtained from the ipsilateral femoral bone marrow were locally applied onto the nerve repair area. Each "treatment" group was further subdivided into two "anti-inflammatory" sub-groups: (1) the with-NSAIDs group, where daily administration of NSAIDs was performed for 7 days post-operatively, and (b) the without-NSAIDs group, where NSAIDs were not administered (Table 1 ).

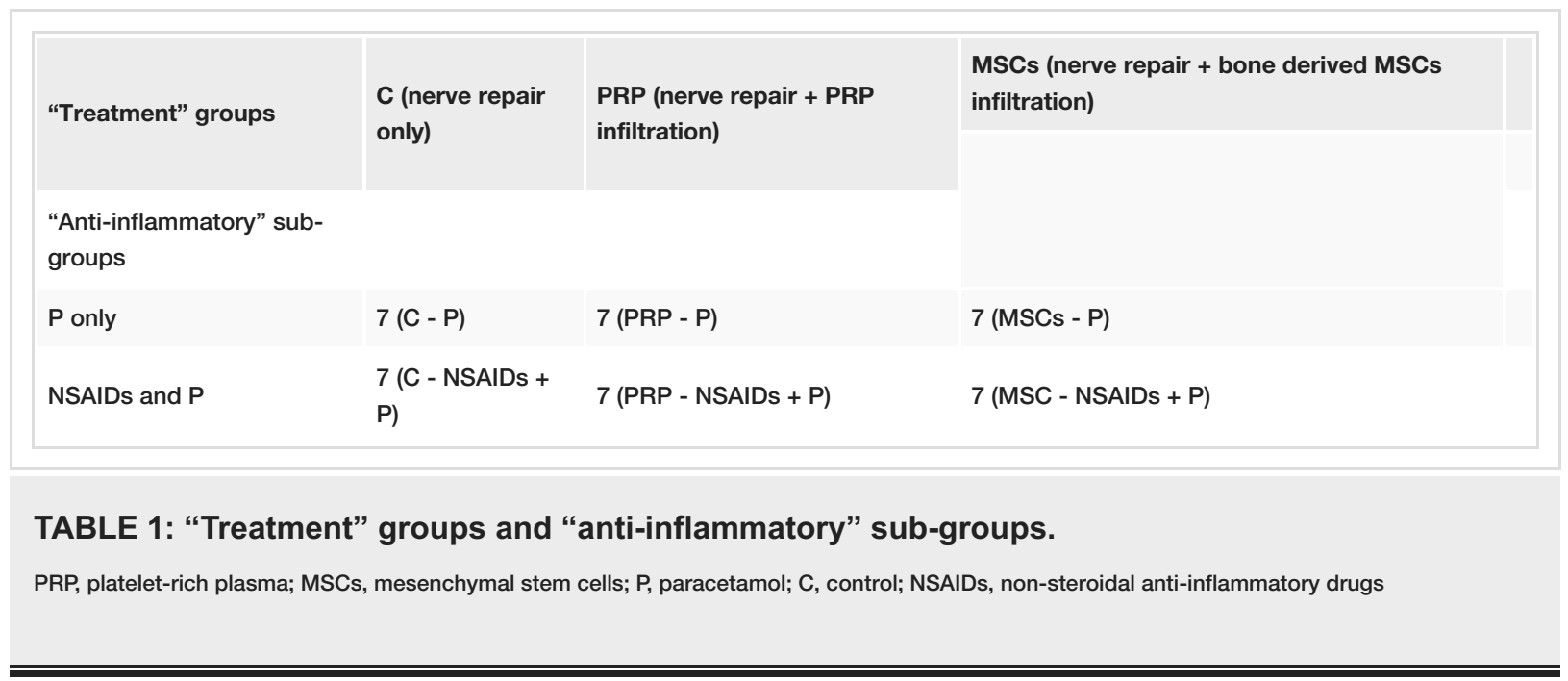

\section{Surgical technique}

Surgery was performed to create the sciatic nerve cut model. A ketamine-medetomidine mixture $(0.5 \mathrm{mg} / \mathrm{kg}$ of medetomidine and $50 \mathrm{mg} / \mathrm{kg}$ of ketamine intramuscularly) was given intraperitoneally for general anesthesia. After the rats were placed on the operation table in the Thompson position, the surgical area was prepped and draped. The sciatic nerve was exposed with an incision that started $1 \mathrm{~cm}$ distal to the sciatic notch and $1 \mathrm{~cm}$ proximal to the trifurcation of the nerve in the posterior part of the knee joint. The sciatic nerve was identified and prepared 3 to $3.5 \mathrm{~cm}$ proximal to its trifurcation division. The nerve was then transversely cut with the use of a micro scalpel $1.5 \mathrm{~cm}$ proximal to the trifurcation (Figures 1,2$)$. Finally, the transected nerve was repaired by the main investigator with epineural sutures (10-0 Ethilon ${ }^{\circledR}$, Ethicon Inc., Somerville, NJ, USA) (Figure 3) under microscopy (x16). In the PRP group, $1.5 \mathrm{~mL}$ of blood drawn from the tail vein was collected in a citrated tube. After 7 minutes of differential centrifugation at $700 \mathrm{RCF}$, the upper portion of the volume $(0.7 \mathrm{~mL}$ of PRP) was injected to an absorbable gelatin sponge $(0.5 \times 0.5 \times 0.1 \mathrm{~cm}$, Spongostan ${ }^{\circledR}$, Ethicon Inc.) and applied onto the repair area. In the MSCs group, MSCs were directly 


\section{Cureus}

collected from the trochanteric area of the femur with the use of a trocar drill. The b-MSCs were then injected onto the absorbable gelatin sponge and applied onto the nerve repair area. In the control group, normal saline $0.09 \%$ was injected onto the absorbable gelatin sponge and applied onto the repair area. The wound was closed with No 4-0 sub-cutaneous absorbable sutures (Vicryl ${ }^{\circledR}$, Ethicon Inc.) and non-absorbable No 5-0 skin sutures (Nylon ${ }^{\circledR}$, Ethicon Inc.). In all rats, a single dose of antibiotic, enrofloxacin (10 mg/kg of Baytril ${ }^{\circledR} 5 \%$, Bayer AG, Leverkusen, Germany), was administered. Following recovery, the rats were returned to their cages and allowed to perform normal activities. All operated animals were again divided in two subgroups: (1) the with-NSAIDs group, where meloxicam ( $0.05 \mathrm{mg} / \mathrm{kg} / 24$ hours) and paracetamol (1 mg/kg/12 hours) was administered for one week, and (b) the without-NSAIDs group, where only paracetamol was administered for one week. At 12 weeks post-operatively, nerve conduction studies were applied to both legs of all the animals. The animals were then sacrificed under anesthesia with bleeding of the posterior vena cava concave vein, and specimens of the repaired sciatic nerves were prepared for histological evaluation.

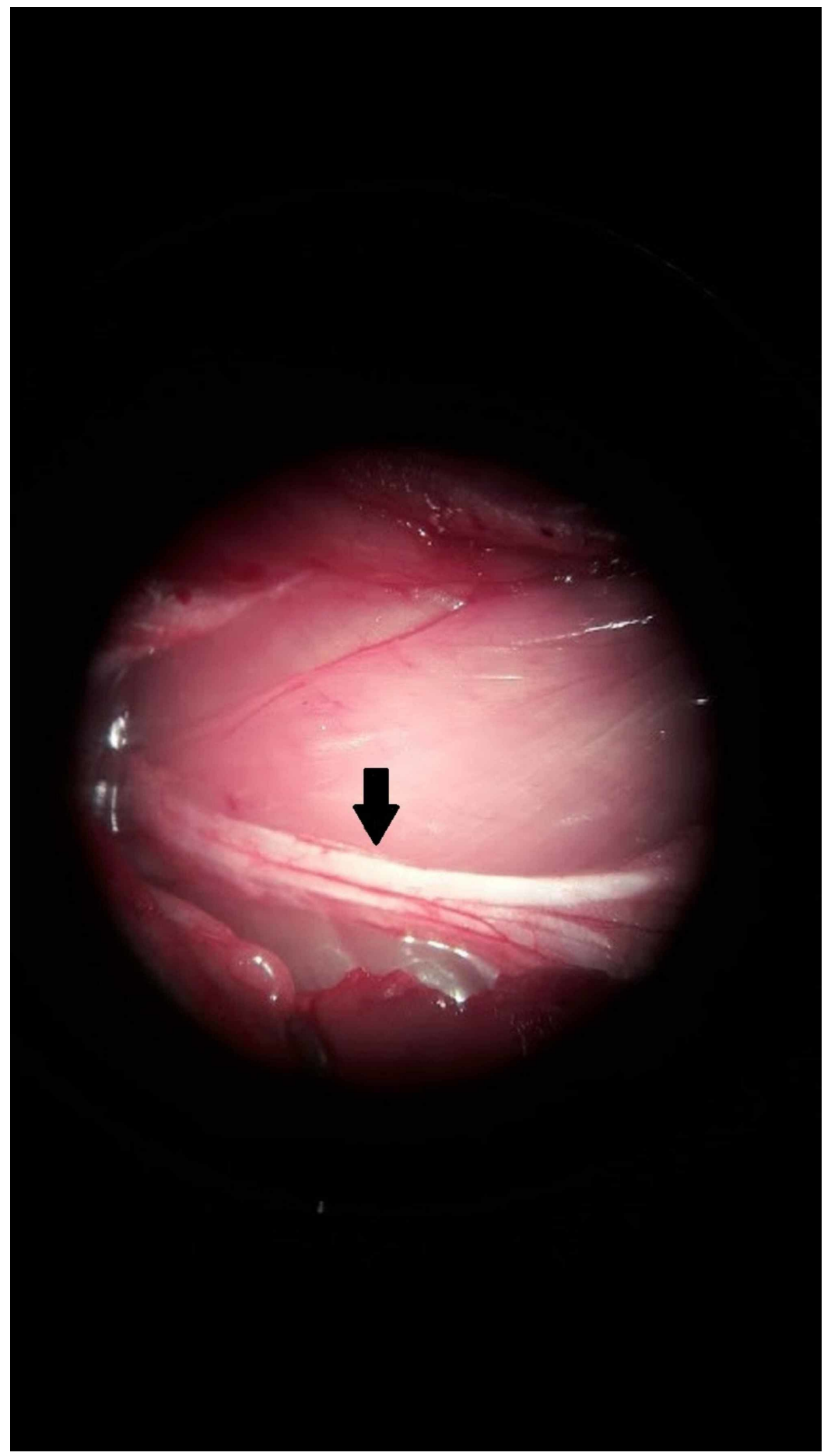

FIGURE 1: Sciatic nerve (black arrow) of the rat before transection (microscopy x16). 


\section{Cureus}

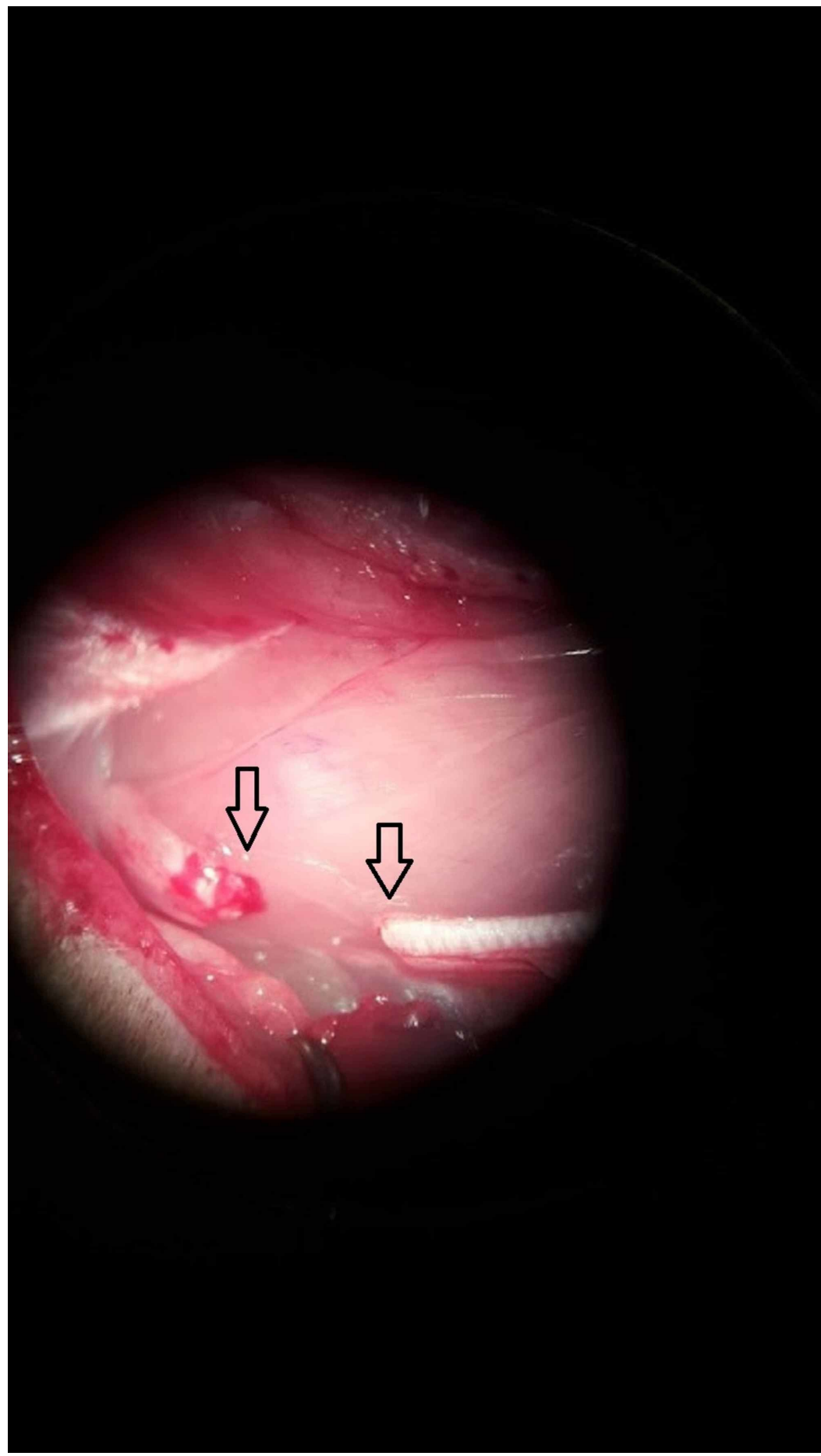

FIGURE 2: Sciatic nerve of the rat after transection (microscopy $x 16$ ). The two arrows show the transected ends. 


\section{Cureus}

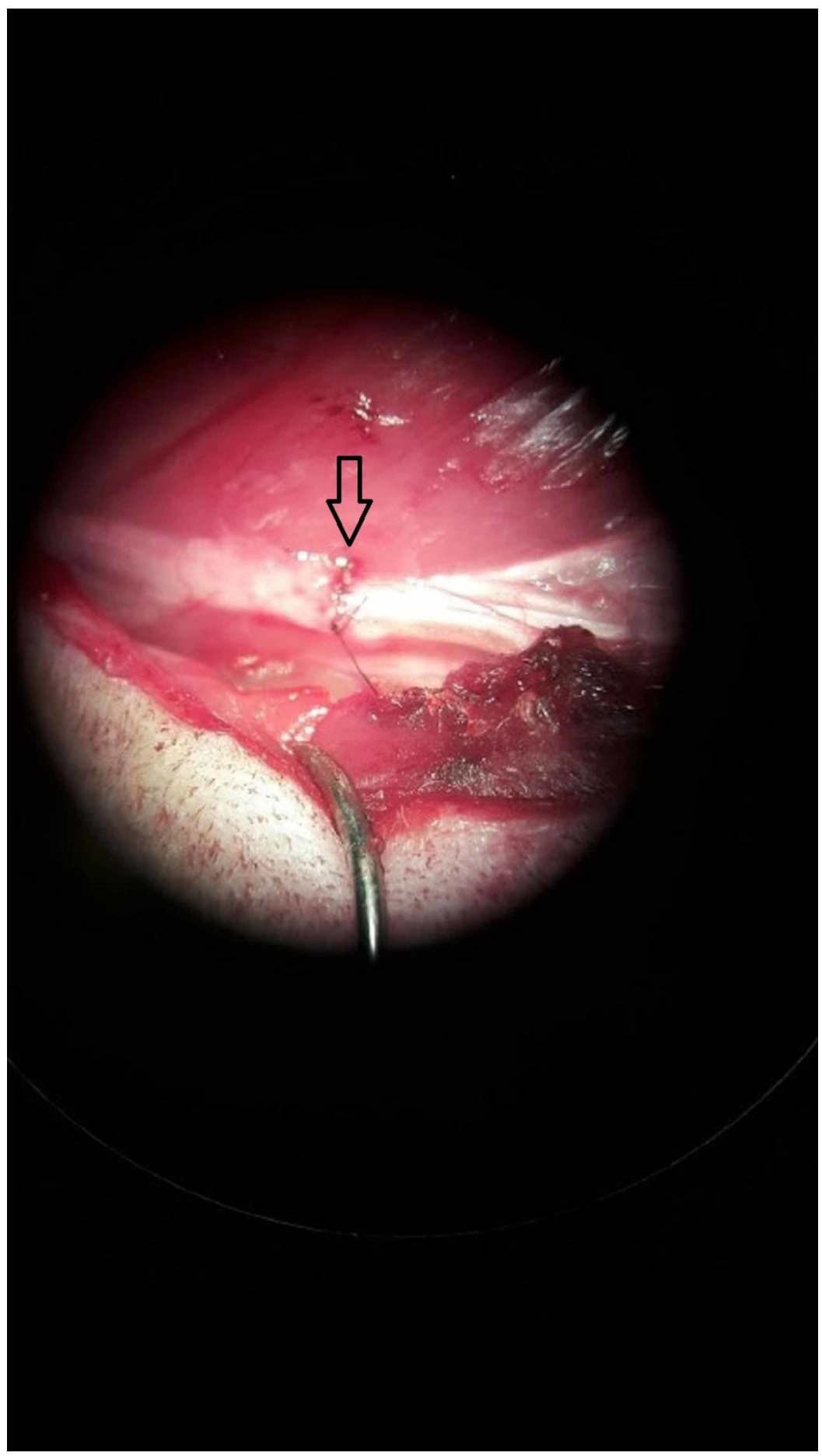

FIGURE 3: Sciatic nerve of the rat after nerve repair (microscopy $\mathbf{x 1 6 ) .}$ The arrow shows the suture repair area.

\section{Nerve conduction study}

All electrodiagnostic evaluations were performed blindly by the same author (P. K.) using the same EMG device. An EMG apparatus (Keypoint ${ }^{\circledR}$ Classic, Medtronics, Copenhagen, Denmark) was used for the measurements. The low- and high-pass filter settings were $20 \mathrm{~Hz}$ and $10 \mathrm{kHz}$, respectively. The stimulus had duration of $0.3 \mathrm{~ms}$, a frequency of $0.5 \mathrm{~Hz}$, and a sweep velocity of $5 \mathrm{~ms} /$ div. Supramaximal stimulation was used. Ring surface electrodes were used for the recordings while the rats were under general anesthesia $(0.5$ $\mathrm{mg} / \mathrm{kg}$ of medetomidine and $50 \mathrm{mg} / \mathrm{kg}$ of ketamine intramuscularly). Subcutaneous platinum needle electrodes (Grass, Astro-Med Inc., West Warwick, RI, USA) were used for stimulation. The sciatic nerve was stimulated supramaximally at the sciatic notch point. Compound muscle action potentials (CMAP) were recorded from the gastrocnemius muscle of both legs. The latency from the stimulus to the first deflection 
from baseline was measured in milliseconds (ms), the CMAP amplitude from the first negative to the next positive peak in millivolts $(\mathrm{mV})$, the CMAP duration from the initial to the terminal deflection back to baseline in $\mathrm{ms}$, and the CMAP area in $\mathrm{ms}{ }^{*} \mathrm{mV}$.

\section{Histological study}

All histology preparation was supervised by the same author (K. D.), and the evaluation was blindly performed by two authors (K. D. and N. K.). The sciatic nerve was fixed in a $10 \%$ formalin buffer solution. Paraffin-embedded tissue was then sectioned into 0.3- to 0.5-mm-thick serial sections on positive charged slides. The sections were stained with hematoxylin-eosin stain and immunohistochemically evaluated with S-100 antibody (polyclonal rabbit) as a marker for myelin sheath (Dako, Glostrup, Denmark). Regarding the S100 antibody, tissues were pre-treated with heat-induced epitope retrieval (HIER) method (PT, Dako) using the Dako Envision Flex Retrieval Solution at high $\mathrm{pH}$ and $96-98^{\circ} \mathrm{C}$ for 20 minutes. The tissue was incubated at room temperature for 30 minutes according to the IHC immunohistochemistry protocol of Dako Envision Flex Kit, which uses diaminobenzidine as chromogen. Samples were photographed under a light microscope (Axioskop 2 Plus, Zeiss, Oberkochen, Germany). Neuronal axons were intensely immunostained with S-100 antigen, and their absolute number was measured [8]. Normal sciatic nerves from the contralateral nonoperated left leg were used as control. The absolute number of axons was measured by examining six randomly selected fields (one center, five periphery) with the use of a digital counter with a x40 magnification from three sites: proximal to nerve repair, distal to nerve repair, and far distal $(0.3 \mathrm{~cm})$ to nerve repair. The procedure was performed both manually under light microscopy and by the ImagePro Plus ${ }^{\circledR}$ v6.0 software interface (Media Cybernetics Inc., Rockville, MD, USA) and applied in the regions of interest for neuronal axons counting and cell morphometry. All evaluations were expressed as a number of positive immunostaining per $1 \mu \mathrm{m}^{2}$.

\section{Statistical analysis}

Data were analyzed by a biostatistician (A. G.) and expressed as mean \pm standard deviation (SD) or mean \pm standard error (SE) (for two-way analysis-of-variance [ANOVA] results) for continuous variables and as percentages for categorical data. The Kolmogorov-Smirnov test was used for normality analysis of the parameters. Two-way ANOVA model was used to examine the interaction between the "treatment" factor and "anti-inflammatory" factor. Since there was no statistically significant interaction, we compared the "treatment" factor regardless of the "anti-inflammatory" factor and the "anti-inflammatory" factor regardless of "treatment" factor.

The comparison of variables factor between "treatment" groups was performed for each sub-group of "antiinflammatory" separately using the one-way ANOVA model. Pairwise comparisons were performed using the Bonferroni test. The comparison of variables between the sub-groups of "anti-inflammatory" factor for each "treatment” group was performed separately using the independent samples t-test.

All tests were two-sided, and statistical significance was set at $\mathrm{p}<0.05$. All analyses were carried out using the statistical package SPSS Version 21 (IBM Corp., Armonk, NY, USA).

\section{Results}

Concerning the CMAP area, there were statistically significant differences between the "treatment" groups $(\mathrm{p}<0.005)$. The PRP group showed a significantly higher CMAP area when compared with the control group $(\mathrm{p}=0.001)$. The MSCs group also demonstrated statistically significant better result when compared with the control group ( $\mathrm{p}=0.006)$. Furthermore, no difference was found between the "anti-inflammatory" subgroups regardless of the "treatment" ( $\mathrm{p}=0.939)$ (Figure 4, Table 2). Two-way ANOVA showed no significant interaction between "treatment" and "anti-inflammatory" factors $(\mathrm{p}=0.704)$. 


\section{Cureus}



FIGURE 4: CMAP area of the operated leg between the "treatment" groups regardless of the "anti-inflammatory" sub-groups.

PRP, platelet-rich plasma; MSCs, mesenchymal stem cells; CMAP, compound muscle action potentials

\begin{tabular}{|c|c|c|c|c|}
\hline & $\begin{array}{l}\text { Non- } \\
\text { NSAIDs } \\
\text { sub-group }\end{array}$ & $\begin{array}{l}\text { NSAIDS } \\
\text { sub-group }\end{array}$ & $\begin{array}{l}\text { Comparison } \\
\text { between sub- } \\
\text { groups }\end{array}$ & $\begin{array}{l}\text { Comparison between "treatment" groups } \\
\text { regardless of "anti-inflammatory" sub- } \\
\text { groups }\end{array}$ \\
\hline & Mean \pm SD & Mean \pm SD & & Mean \pm SE \\
\hline Control & $4.62 \pm 3.28^{a}$ & $3.37 \pm 2.68^{b}$ & $p=0.46 /$ & $3.99 \pm 1.38^{b}$ \\
\hline MSCs & $4.88 \pm 3.54^{\mathrm{a}}$ & $6.60 \pm 5.01^{a}$ & $p=0.463$ & $5.74 \pm 1.34^{b}$ \\
\hline PRP & $12.52 \pm 8.79$ & $11.68 \pm 4.94$ & $\mathrm{p}=0.823$ & $12.10 \pm 1.34$ \\
\hline \multirow[t]{2}{*}{ Comparison between “treatment” groups } & $p=0.036$ & $p=0.006$ & & $p<0.0005$ \\
\hline & Mean \pm SE & Mean \pm SE & & \\
\hline $\begin{array}{l}\text { Comparison between "anti-inflammatory" } \\
\text { sub-groups regardless of "treatment" } \\
\text { groups }\end{array}$ & $7.34 \pm 1.12$ & $6.21 \pm 1.10$ & $\mathrm{p}=0.939$ & $\begin{array}{l}\text { Interaction between "treatment groups and } \\
\text { "anti-inflammatory" sub-groups, } p=0.704\end{array}$ \\
\hline
\end{tabular}

\section{TABLE 2: CMAP area $(\mathrm{mV} / \mathrm{msec})$ of the operated leg.}

${ }^{a} p<0.05$ vs. the PRP group. ${ }^{b} p<0.005$ vs. the PRP group.

NSAIDs, non-steroidal anti-inflammatory drugs; SD, standard deviation; SE, standard error; MSCs, mesenchymal stem cells; PRP, platelet-rich plasma; CMAP, compound muscle action potentials

With respect to the CMAP ratio area\% (operated/non-operated*100), there was a statistically significant difference between "treatment" groups ( $\mathrm{p}=0.005$ ). PRP group showed statistically significant better results when compared with the control group $(\mathrm{p}=0.003)$. Furthermore, no difference was found between the "antiinflammatory" sub-groups regardless of "treatment" ( $p=0.404)$ (Figure 5, Table 3). Two-way ANOVA showed no significant interaction between the "treatment" and "anti-inflammatory" factors $(\mathrm{p}=0.477)$. 


\section{Cureus}

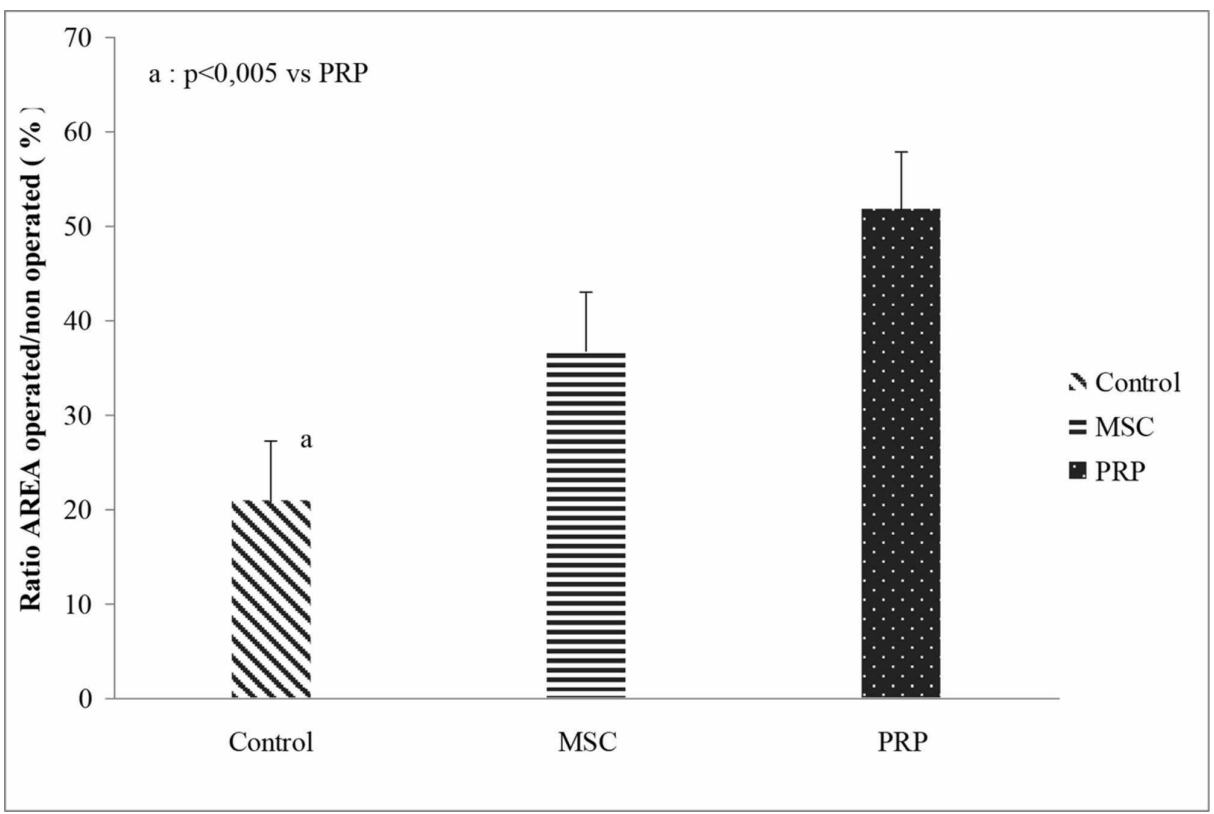

FIGURE 5: CMAP area ratio (operated leg / non-operated leg *100) between the "treatment" groups regardless of the "anti-inflammatory" sub-groups.

PRP, platelet-rich plasma; MSCs, mesenchymal stem cells; CMAP, compound muscle action potentials

\begin{tabular}{|c|c|c|c|c|}
\hline & $\begin{array}{l}\text { Non- } \\
\text { NSAIDs } \\
\text { sub-groups }\end{array}$ & $\begin{array}{l}\text { NSAIDS sub- } \\
\text { groups }\end{array}$ & $\begin{array}{l}\text { Comparison } \\
\text { between sub- } \\
\text { groups }\end{array}$ & $\begin{array}{l}\text { Comparison between "treatment" } \\
\text { groups regardless of "anti- } \\
\text { inflammatory" sub-groups }\end{array}$ \\
\hline & Mean $\pm S D$ & Mean $\pm S D$ & & Mean \pm SE \\
\hline Control & $22.82 \pm 19.70$ & $19.07 \pm 17.66^{a}$ & $p=0.724$ & $20.94 \pm 6.26^{b}$ \\
\hline MSCs & $28.18 \pm 11.16$ & $45.61 \pm 34.27$ & $p=0.277$ & $36.89 \pm 6.07$ \\
\hline PRP & $49.65 \pm 24.42$ & $53.91 \pm 24.42$ & $p=0.752$ & $51.78 \pm 6.07$ \\
\hline \multirow[t]{2}{*}{ Comparison between "treatment" groups } & $p=0.161$ & $p=0.047$ & & $p=0.005$ \\
\hline & Mean \pm SE & Mean \pm SE & & \multirow{2}{*}{$\begin{array}{l}\text { Interaction between "treatment" groups } \\
\text { and "anti-inflammatory" sub-groups, } \\
p=0.477\end{array}$} \\
\hline $\begin{array}{l}\text { Comparison between "anti-inflammatory" } \\
\text { sub-groups regardless of "treatment" } \\
\text { groups }\end{array}$ & $33.55 \pm 5.08$ & $39.53 \pm 4.94$ & $\mathrm{p}=0.404$ & \\
\hline
\end{tabular}

\section{TABLE 3: Ratio of CMAP area between the operated leg and the non-operated leg.}

${ }^{a} p<0.05$ vs. the PRP group. ${ }^{b} p<0.005$ vs. the PRP group.

NSAIDs, non-steroidal anti-inflammatory drugs; SD, standard deviation; SE, standard error; MSCs, mesenchymal stem cells; PRP, platelet-rich plasma; CMAP, compound muscle action potentials

As regards the CMAP duration, no significant differences were found between the "treatment" groups. However, the CMAP amplitude of the right gastrocnemius muscle was significantly higher for the PRP group when compared with the control group but not significant when compared with the non-operated (intact) left leg.

The absolute number of neural axons (number/ $\mu^{2}{ }^{2}$ ) distal to nerve repair was significantly higher in PRP group when compared to the control group $(\mathrm{p}=0.005)$. However, it was not the same in the MSCs group when 


\section{Cureus}

compared with the PRP group (Figure 6).

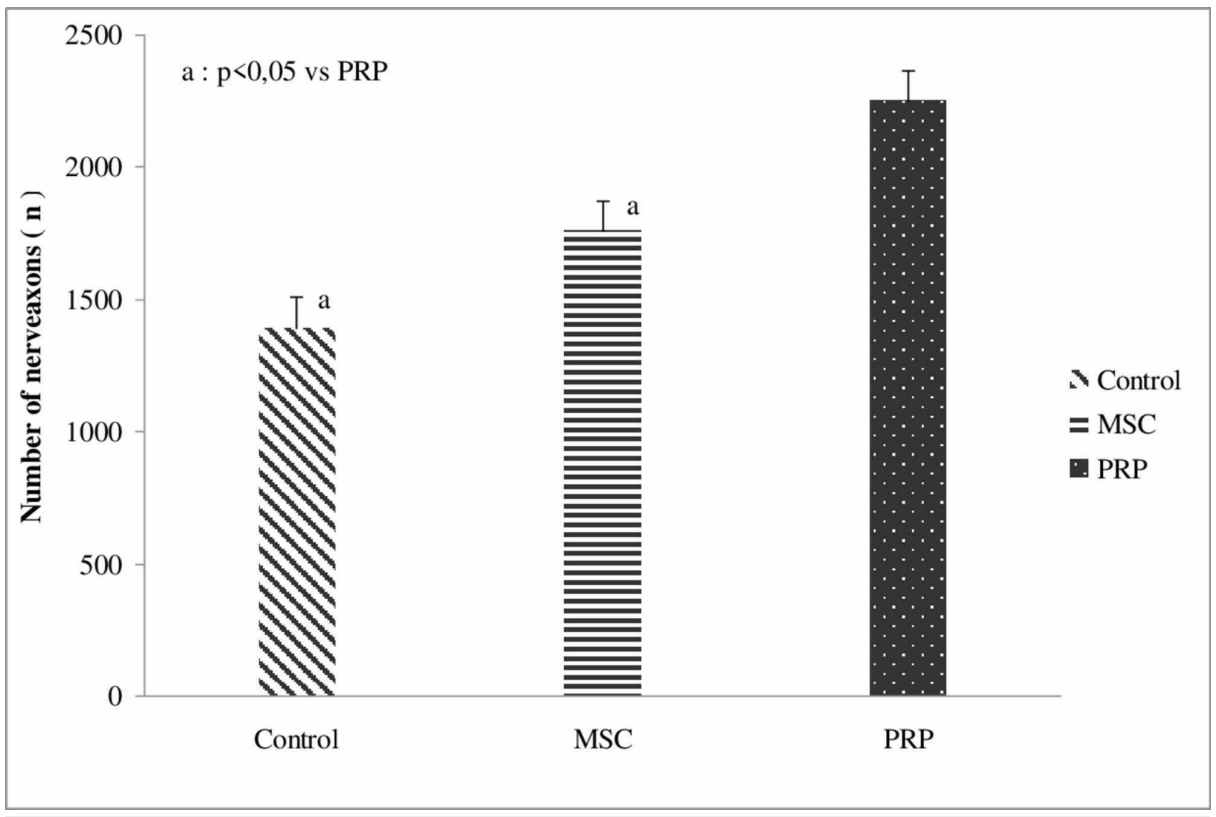

FIGURE 6: Absolute number of nerve axons distal to nerve repair between the "treatment" groups regardless of the "anti-inflammatory" sub-groups.

PRP, platelet-rich plasma; MSCs, mesenchymal stem cells

The ratio of the number of axons distal to nerve repair and the number of axons proximal to nerve repair (D/P) was significantly higher in the PRP and MSCs groups when compared with the control group $(\mathrm{p}=0.009$ and $\mathrm{p}=0.047$, respectively). No difference was found between the "anti-inflammatory" sub-groups regardless of the treatment (Figure 7, Table 4). The two-way ANOVA result showed no significant interaction between the "treatment" and "anti-inflammatory factors" ( $\mathrm{p}=0.822)$.



FIGURE 7: DP ratio of the average number of neuronal axons between the "treatment" groups regardless of the "anti-inflammatory" subgroups.

PRP, platelet-rich plasma; MSCs, mesenchymal stem cells; DP, distal nerve repair to proximal nerve repair 


\section{Cureus}

\begin{tabular}{|c|c|c|c|c|}
\hline & $\begin{array}{l}\text { Non- } \\
\text { NSAIDs } \\
\text { sub-groups }\end{array}$ & $\begin{array}{l}\text { NSAIDs } \\
\text { sub-groups }\end{array}$ & $\begin{array}{l}\text { Comparison } \\
\text { between sub- } \\
\text { groups }\end{array}$ & $\begin{array}{l}\text { Comparison between "treatment" } \\
\text { groups regardless of "anti- } \\
\text { inflammatory" sub-groups }\end{array}$ \\
\hline & Mean $\pm S D$ & Mean $\pm S D$ & & Mean \pm SE \\
\hline Control & $62.76 \pm 12.42$ & $71.14 \pm 16.50$ & $p=0.331$ & $66.9 \pm 4.4$ \\
\hline MSCs & $79.97 \pm 19.80$ & $85.16 \pm 15.53$ & $p=0.606$ & $82.6 \pm 4.3^{\mathrm{a}}$ \\
\hline PRP & $86.28 \pm 15.95$ & $86.98 \pm 12.96$ & $p=0.929$ & $86.7 \pm 4.3^{b}$ \\
\hline Comparison between "treatment" groups & $\mathrm{p}=0.066$ & $p=0.119$ & & $\mathrm{p}=0.007$ \\
\hline $\begin{array}{l}\text { Comparison between "anti-inflammatory" } \\
\text { sub-groups regardless of "treatment" } \\
\text { groups }\end{array}$ & $76.3 \pm 3.58$ & $81.1 \pm 3.48$ & $\mathrm{p}=0.348$ & $\begin{array}{l}\text { Interaction between "treatment" groups } \\
\text { and "anti-inflammatory" sub-groups, } \\
\mathrm{p}=0.822\end{array}$ \\
\hline
\end{tabular}

\section{TABLE 4: DP ratio.}

${ }^{a} p=0.047$ vs. the control group. ${ }^{b} p=0.009$ vs. the control group.

With regard to the diameter of the newly formed neuroaxons, there was no significant difference between the "treatment" groups or the "anti-inflammatory" sub-groups (Figure 8). 


\section{Cureus}

Proximal Nerve Repair

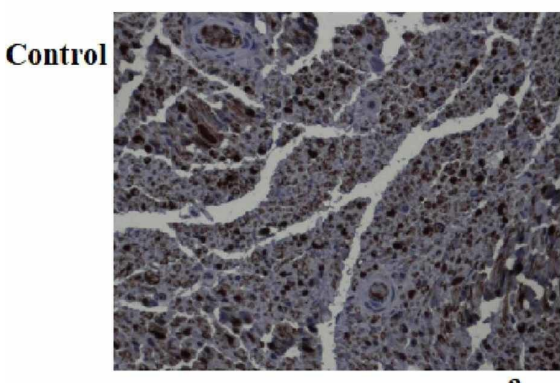

a

PRP

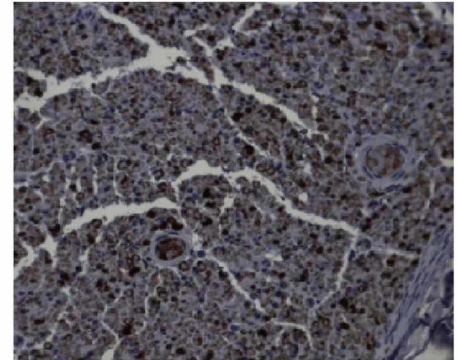

c

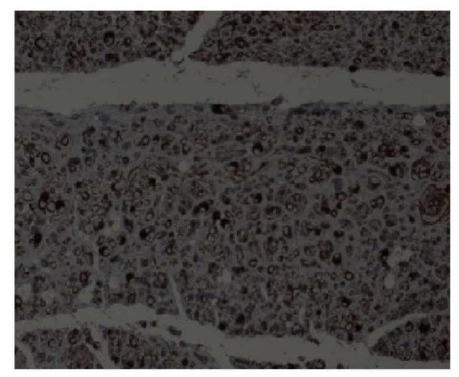

Distal Nerve Repair
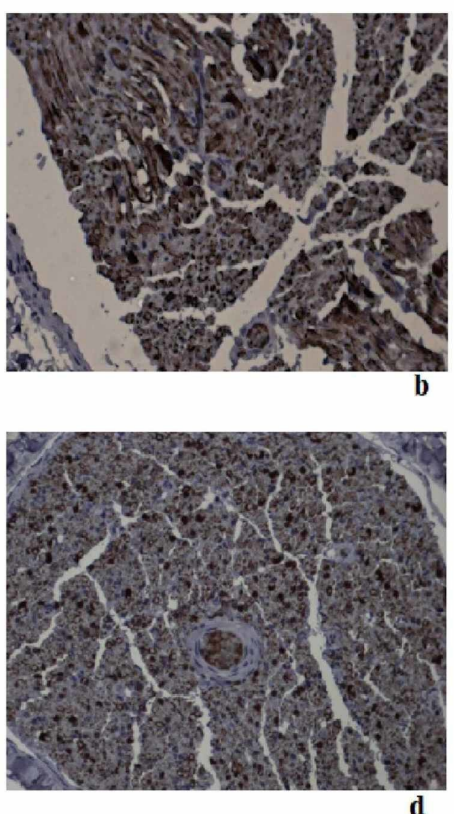

d

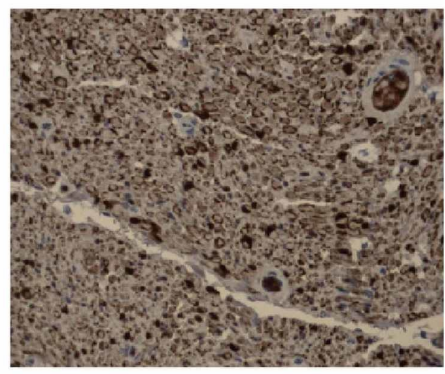

$\mathrm{f}$

FIGURE 8: Histology under $\mathbf{x 4 0}$ magnification proximal and distal to nerve repair of the three "treatment" groups.

(a) Control group proximal to nerve repair. (b) Control group distal to nerve repair. (c) PRP group proximal to nerve repair. (d) PRP group distal to nerve repair. (e) MSCs group proximal to nerve repair. (f) MSCs group distal to nerve repair.

PRP, platelet-rich plasma; MSCs, mesenchymal stem cells

\section{Discussion}

Microsurgical suturing techniques for fine nerve repair have witnessed impressive development in recent years. Furthermore, bio-artificial structures called "conduits" serve as an alternative to autologous nerve grafts, with the aim to bridge the defects in nerve discontinuity [9]. However, these methods failed to constantly reproduce satisfactory results for nerve repair [2]. Despite extensive studies in the field of nerve regeneration, the molecular mechanisms underlying the post-traumatic processes in nerve fibers remain poorly understood and require further investigation [10]. Adjuvant biologic treatment with local application of PRP or MSCs on the nerve repair site remains a wide-open field for research. The results of the present experimental animal study showed that enrichment of PRP or MSCs on nerve repair resulted in better 
functional recovery of the sciatic nerve with better structural indexes on regenerated nerve mainly on the distal part of the repair, indicating that biological adjuvant therapies may play a role in the peripheral nerve regeneration.

Ding et al. in their study of 24 rats applied local PRP after creating crush injury on the cavernous nerve [11]. The authors concluded that the functional healing and histological parameters in the PRP group were significantly better than that in the placebo group. A similar study in which a crush injury was created on the sciatic rat nerve also showed positive effects of PRP on nerve regeneration [12]. Sariguney et al. concluded that PRP is effective when applied following the ideal surgical nerve repair in a nerve transection model and was ineffective in cases of insufficient surgical repair [13]. In contrast to the above positive effects of PRP, recent studies reported unsatisfactory results. Welch et al. reported no significant effect of PRPs on a transection and direct repair on a rat model [14]. Piskin et al. concluded that PRPs did not improve nerve regeneration after microsurgical reconstruction of a nerve gap using collagen tubes [4]. As a cellular carrier, two studies in an acute nerve injury model in guinea pigs and rabbits applied PRPs and seeded the acellular carrier with MSCs, and reported beneficial effects on axonal counts, myelination, and electrophysiological parameters [15,16]. PRPs have also been used as a filler of acellular nerve allografts [17]. They showed a dose-dependent effect on the proliferation, migration, and neurotrophic function in rat MSCs cultured with PRP. They also showed significant improvement in the diameter, thickness, and number of myelinating axons, as well as an enhancement of electrophysiological parameters in sciatic nerve injury repaired with autografts and PRP in a rat model [18].

Using platelet-rich fibrin (PRF) as a filler of silicon nerve guidance or nerve grafts in a rat model, animals treated with PRPs showed improved functional recovery and a superior sciatic functional index compared with non-treated animals [19,20]. However, the researchers did not find morphometric or structural improvements. The application of PRPs as a fibrin membrane to wrap the neurorrhaphy in an acute injury model of sciatic nerve neurotmesis showed diverse positive effects [21]. The authors observed a stronger EMG signal, a significantly larger axonal density, and a lower scar tissue in animals treated with PRP fibrin membranes, and remains of PRP membranes were still present after six weeks post-surgery. In this sense, two studies reported the positive effects of using PRP as adjuvant treatment in nerve suturing. Farrag et al. reported that PRPs may enhance the myelin thickness and increase the axon counts when the injured nerve is sutured and assisted with PRP, whereas Sariguney et al. found no positive effects on axonal size in sutured nerves enriched with PRP. However, they showed a better functional outcome associated with improvement in the myelin thickness and the onset latency [13,22].

Regenerative cell therapy pathways that influence a successful outcome after a nerve repair are still poorly understood. These biological mechanisms could be classified into the following groups: (1) differentiation toward the Schwann cell lineage, (2) contribution to myelination of regenerating axons, (3) production of trophic factors and extracellular matrix proteins that provide a milieu for axonal outgrowth, (4) stimulation of proliferation and differentiation of endogenous cells, (5) stimulation of angiogenesis, and (6) immunosuppression. The analysis of the molecular mechanisms underlying the interaction between MSCs and immune cells demonstrated that MSCs suppress T and B lymphocytes and inhibit dendrite cell maturation [23]. MSCs enhances axon regeneration not only when delivered to the injured nerve or conduit bridging the nerve gap but also when administered intravenously [6,7]. Furthermore, Tomita et al. performed in vitro and in vivo studies of glial differentiation of MSCs derived from human adipose tissue. It was established that following exposure to glial growth factors, MSCs transdifferentiate into a Schwann cell phenotype. Lineage-committed MSCs demonstrated a seven-fold higher survival rate after implantation than multipotent MSCs in a rat tibial nerve injury study [24].

Non-steroid anti-inflammatory drugs (NSAIDs) administration seems to positively affect nerve regeneration. Madura et al. showed that ibuprofen significantly enhanced regeneration after tibial nerve axonotomy and repair in a rat model [25]. Sharp et al. showed that their results only partially replicate the findings that the treatment with ibuprofen improves motor function after spinal cord injury but failed to replicate findings regarding enhanced axon growth [26]. Moreover, an immunohistochemical study showed a more positive location of reactions to S-100 in the group loaded with diclofenac in an artery graft than the group in which an artery graft was buffered with saline alone. Therefore, the diclofenac improved functional recovery and morphometric indices of the sciatic nerve [8]. However, in our study, despite current literature, systematic NSAIDs administration did not show any additional positive effect on nerve regeneration.

\section{Limitations}

There were a few limitations in this study. We performed a single centrifugation process and produced leukocyte-rich PRPs rather than pure PRP (p-PRP). The b-MSCs were immediately applied to the nerve repair site instead of culturing them up to a high concentration of pure MSCs. However, as mentioned earlier, in clinical practice when a nerve injury is diagnosed, a nerve repair is an urgent situation and there is no time for p-PRP preparation or MSCs cultivation. Advantages of our study were (1) the use of contralateral leg to adjust the EMG values between injured and non-injured leg for each animal and (2) the use of a statistically significant number of subjects following power analysis of the protocol. 


\section{Conclusions}

In this study, the intra-operative administration of PRP or MSCs following a sciatic nerve repair improved histology architecture and enhanced functional outcome compared to the control groups. The results of this study are encouraging for clinical trials in humans supporting the findings of other recent studies. Local intra-operative single-dose administration of either PRP or MSCs on the repair site of a damaged nerve seems to enhance tissue regeneration in terms of histological findings and therefore may ameliorate the final functional outcome in terms of EMG findings. In clinical practice, the healing process of an acutely repaired peripheral nerve may benefit from adjuvant biological therapy. Thus, the intra-operative use of autologous biological substances such as PRPs obtained from peripheral veins or MSCs harvested from cancellous bone marrow is a safe, low-cost, fast, single-stage technique that may enhance the nerve reparative procedure and the final functional outcome.

\section{Additional Information \\ Disclosures}

Human subjects: All authors have confirmed that this study did not involve human participants or tissue. Animal subjects: Directorate of Veterinary Services of Prefecture of Athens, Attica, Greece, according to Greek legislation regarding ethical and experimental procedures. Issued protocol number EL 25 BIO 018. Conflicts of interest: In compliance with the ICMJE uniform disclosure form, all authors declare the following: Payment/services info: All authors have declared that no financial support was received from any organization for the submitted work. Financial relationships: All authors have declared that they have no financial relationships at present or within the previous three years with any organizations that might have an interest in the submitted work. Other relationships: All authors have declared that there are no other relationships or activities that could appear to have influenced the submitted work.

\section{References}

1. Fowler JR, Lavasani M, Huard J, Goitz RJ: Biologic strategies to improve nerve regeneration after peripheral nerve repair. J Reconstr Microsurg. 2015, 31:243-248. 10.1055/s-0034-1394091

2. Faroni A, Mobasseri SA, Kingham PJ, Reid AJ: Peripheral nerve regeneration: experimental strategies and future perspectives. Adv Drug Deliv Rev. 2015, 82:160-167. 10.1016/j.addr.2014.11.010

3. Anitua E, Pascual C, Perez-Gonzalez R, Antequera D, Padilla S, Orive G, Carro E: Intranasal delivery of plasma and platelet growth factors using PRGF-Endoret system enhances neurogenesis in a mouse model of Alzheimer's disease. PLoS One. 2013, 8:73-118. 10.1371/journal.pone.0073118

4. Piskin A, Kaplan S, Aktaş A, et al.: Platelet gel does not improve peripheral nerve regeneration: an electrophysiological, stereological, and electron microscopic study. Microsurgery. 2009, 29:144-153. 10.1002/micr.20599

5. McGrath AM, Brohlin M, Kingham PJ, Novikov LN, Wiberg M, Novikova LN: Fibrin conduit supplemented with human mesenchymal stem cells and immunosuppressive treatment enhances regeneration after peripheral nerve injury. Neurosci Lett. 2012, 16:171-176. 10.1016/j.neulet.2012.03.041

6. Fairbairn NG, Meppelink AM, Ng-Glazier J, Randolph MA, Winograd JM: Augmenting peripheral nerve regeneration using stem cells: a review of current opinion. World J Stem Cells. 2015, 7:11-26. 10.4252/wjsc.v7.i1.11

7. Marconi S, Castiglione G, Turano E, et al.: Human adipose-derived mesenchymal stem cells systemically injected promote peripheral nerve regeneration in the mouse model of sciatic crush. Tissue Engineering. 2012, 18:1264-1272. 10.1089/ten.TEA.2011.0491

8. Rahim M, Hirsaee MA, Amini K: Improvement of functional recovery of transected peripheral nerve by means of artery grafts filled with diclofenace. Int J Surg. 2013, 11:259-264. 10.1016/j.ijsu.2013.01.008

9. Saltzman EB, Villa JC, Doty SB, Feinberg JH, Lee SK, Wolfe SW: A comparison between two collagen nerve conduits and nerve autograft: a rat model of motor nerve regeneration. J Hand Surg Am. 2019, 44:700-701. 10.1016/j.jhsa.2018.10.008

10. Chen P, Piao X, Bonaldo P: Role of macrophages in Wallerian degeneration and axonal regeneration after peripheral nerve injury. Acta Neuropathol. 2015, 130:605-618. 10.1007/s00401-015-1482-4

11. Ding XG, Li SW, Zheng XM, Hu LQ, Hu WL, Luo Y: The effect of platelet-rich plasma on cavernous nerve regeneration in a rat model. Asian J Androl. 2009, 11:215-221. 10.1038/aja.2008.37

12. Emel E, Ergun SS, Kotan D, Gürsoy EB, Parman Y, Zengin A, Nurten A: Effects of insulin-like growth factor-I and platelet-rich plasma on sciatic nerve crush injury in a rat model. J Neurosurg. 2011, 114:522-528. 10.3171/2010.9.JNS091928

13. Sariguney Y, Yavuzer R, Elmas C, Yenicesu I, Bolay H, Atabay K: Effect of platelet-rich plasma on peripheral nerve regeneration. J Reconstr Microsurg. 2008, 24:159-167. 10.1055/s-2008-1076752

14. Welch JA, Kraus KH, Wells MR, Blunt DG, Weremowitz J: Effect of combined administration of insulinlikegrowth factor and platelet-derived growth factor on theregeneration of transected and anastomosed sciatic nervein rats. Am J Vet Res. 1997, 58:1033-1037.

15. Cho HH, Jang S, Lee SC, et al.: Effect of neural-induced mesenchymal stem cells and platelet-rich plasma on facial nerve regeneration in an acute nerve injury model. Laryngoscope. 2010, 120:907-913. 10.1002/lary.20860

16. Yu W, Wang J, Yin J: Platelet-rich plasma: a promising product for treatment of peripheral nerve regeneration after nerve injury. Int J Neurosci. 2011, 121:176-180. 10.3109/00207454.2010.544432

17. Zheng C, Zhu Q, Liu X, Huang X, He C, Jiang L, Quan D: Effect of platelet-rich plasma (PRP) concentration on proliferation, neurotrophic function and migration of Schwann cells in vitro. J Tissue Eng Regen Med. 2016, 10:428-436. 10.1002/term.1756 
18. Zheng C, Zhu Q, Liu X, Huang X, He C, Jiang L, Quan D: Improved peripheral nerve regeneration using acellular nerve allografts loaded with platelet-rich plasma. Tissue Eng Part A. 2014, 20:3228-3240. 10.1089/ten.TEA.2013.0729

19. Lichtenfels M, Colomé L, Sebben AD, Braga-Silva J: Effect of platelet rich plasma and platelet rich fibrin on sciatic nerve regeneration in a rat model. Microsurgery. 2013, 33:383-390. 10.1002/micr.22105

20. Sabongi RG, De Rizzo LALM, Fernandes M, Valente SG, Gomes dos Santos JB, Faloppa F, Leite VM: Nerve regeneration: is there an alternative to nervous graft?. J Reconstr Microsurg. 2014, 30:607-616. 10.1055/s0034-1372477

21. Giannessi E, Coli A, Stornelli MR, et al.: An autologously generated platelet-rich plasma suturable membrane may enhance peripheral nerve regeneration after neurorraphy in an acute injury model of sciatic nerve neurotmesis. J Reconstr Microsurg. 2014, 30:617-626. 10.1055/s-0034-1372483

22. Farrag TY, Lehar M, Verhaegen P, Carson KA, Byrne PJ: Effect of platelet rich plasma and fibrin sealant on facial nerve regeneration in a rat model. Laryngoscope. 2007, 117:157-165. 10.1097/01.mlg.0000249726.98801.77

23. Rubtsov YP, Suzdaltseva YG, Goryunov KV, Kalinina NI, Sysoeva VY, Tkachuk VA: Regulation of immunity via multipotent mesenchymal stromal cells. Acta Naturae. 2012, 4:23-31.

24. Tomita K, Madura T, Mantovani C, Terenghi G: Differentiated adipose-derived stem cells promote myelination and enhance functional recovery in a rat model of chronic denervation. J Neurosci Res. 2012, 90:1392-1402. 10.1002/jnr.23002

25. Madura T, Tamita K, Terenghi G: Ibuprofen improves functional outcome after axotomy and immediate repair in the peripheral nervous system. J Plast Reconstr Aesthet Surg. 2011, 64:1641-1646. 10.1016/j.bjps.2011.07.014

26. Sharp KG1, Yee KM, Stiles TL, Aguilar RM, Steward O: A re-assessment of the effects of treatment with a non-steroidal anti-inflammatory (ibuprofen) on promoting axon regeneration via RhoA inhibition after spinal cord injury. Exp Neurol. 2013, 248:321-337. 10.1016/j.expneurol.2013.06.023 